International Journal of Pure and Applied Mathematics

Volume 99 No. 2 2015, 165-175

ISSN: 1311-8080 (printed version); ISSN: 1314-3395 (on-line version)

url: http://www.ijpam.eu

doi: http://dx.doi.org/10.12732/ijpam.v99i2.4

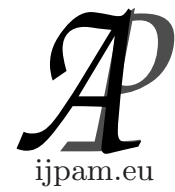

\title{
GENERALIZED BANACH CONTRACTION PRINCIPLE IN INCLOSED SET
}

\author{
Prasanta Kumar Das ${ }^{1}$, Susanta Kumar Mohanta ${ }^{2}$ \\ ${ }^{1,2}$ Department of Mathematics \\ School of Applied Sciences \\ KIIT University \\ Bhubaneswar, 751024, INDIA
}

AMS Subject Classification: 65K10, 90C33, 47J30

Key Words: invex set, inclosed set, generalized variational inequality, generalized complementarity problems, generalized Banach contraction principle

\section{Introduction}

The study of variational inequalities is a part of development in the theory of optimization theory because optimization problems can often be reduced to the solution of variational inequalities. In 1964, Stampacchia [10] introduced the concept of variational inequality problem to study the problems arises in various fields such as optimization, physical sciences, Engineering studies, Economics etc. Several authors have proved many fascinating results on variational inequality problem. We list some of them, which are used frequently in this paper. The existence of the solution to the problem is studied by many authors such as, M. Chipot [2], J.L. Lions and G. Stampacchia [8], R.W. Cottle, F. Giannessi and J.L. Lions [3], etc. to name only a few.

During the study of existence theorems of the variational inequality problems, the property of the domain (such as: closedness, convexity, boundedness etc.) plays an important role. For our study we first establish the existence of invariant closed set with respect to a vector function $\eta$ (in short: $\eta$-closed set)

Received: October 13, 2014

(c) 2015 Academic Publications, Ltd.

$\S$ Correspondence author url: www.acadpubl.eu 
in Hilbert space and a $x^{*}$-generalized variational inequality problem is studied in it. Next we prove the existence theorem of generalized Banach contraction principle to study the $x^{*}$-generalized complementarity problem in Hilbert space.

\section{Inclosed set and its existence theorems}

For our need, we recall the following definition.

Definition 2.1. [6] The set $K$ is said to be $\eta$-invex set if there exist a vector function $\eta: K \times K \rightarrow X$ such that for all $x, y \in K$ and $t \in(0,1)$, we have

$$
y+\operatorname{t\eta }(x, y) \in K
$$

The concept of invariant closed set (or inclosed set) with respect to $\eta$ (in short: $\eta$-closed set) is defined as follows.

Definition 2.2. [4] The set $X$ is said to be invariant closed set (or inclosed) with respect to $\eta$, i.e., $\eta$-closed set if for every $y \in X$, there is a unique $x \in X$ closest to $p \in \mathbb{H}$ with respect to $\eta$, that is,

$$
\langle x, \eta(z, x)\rangle \geq\langle p, \eta(z, x)\rangle \quad \text { for all } z \in X
$$

in other words, for every $p \in \mathbb{H}$, there is a unique $x \in X$ closest to $p$ with respect to $\eta$.

Remark 2.3. If $\eta(z, x)=z-x$, then $X$ is closed.

Das and Mohanta have studied the existence theorem of $\eta$-closed set as follows.

Theorem 2.4. [4] Let $X$ be any subset of the Hilbert space $\mathbb{H}$. Let $\eta: X \times X \rightarrow \mathbb{H}$ be any continuous map. If for every $p \in \mathbb{H}$, there is a unique $x \in X$ such that for all $z \in X$, for any $t \in(0,1)$, we have

(i) $\|x-\operatorname{t\eta }(z, x)-p\| \leq\|z-t \eta(z, x)-p\|$,

(ii) $2 t\langle p-z, \eta(z, x)\rangle \leq 2\langle p, z-x\rangle+\langle x, x\rangle-\langle z, z\rangle$.

Then $X$ is $\eta$-closed. 
In the following theorem we show the alternative theorem for $\eta$-closedness of any set.

Theorem 2.5. Let $X$ be a nonempty closed $\eta$-invex set in $\mathbb{H}$, where $\eta: X \times X \rightarrow \mathbb{H}$ is any map. Then $X$ is inclosed with respect to $\eta$, i.e., $\eta$-closed.

Proof. $X$ be a nonempty closed set in $\mathbb{H}$, so for every $y \in X$, there is a unique $x \in X$ closest to $p \in H$ with respect to $\eta$, i.e.,

$$
\|x-p\| \leq\|z-p\|
$$

for every $z \in X$. Let $z$ be any point of $X$ and $t \in[0,1]$. Since $X$ is $\eta$-invex, $x+t \eta(z, x) \in X$ for all $x, z \in X$. Define the function

$$
\phi:[0,1] \rightarrow \mathbb{R}_{+}
$$

that corresponds to $x$ with respect to $\eta$ by the rule

$$
\phi(t)=\|p-x-t \eta(z, x)\|^{2}
$$

for all $x, z \in X$. Then $\phi$ is twice differentiable function of $t$ and

$$
\phi^{\prime}(t)=2\langle p-x-t \eta(z, x),-\eta(z, x)\rangle=-2\langle p-x-t \eta(z, x), \eta(z, x)\rangle
$$

for all $z \in X$. Since $x$ is the unique element closest to $p$, we must have

$$
\phi^{\prime}(0) \geq 0
$$

and therefore

$$
-\langle p-x, \eta(z, x)\rangle \geq 0
$$

for all $z \in X$, implying

$$
\langle x, \eta(z, x)\rangle \geq\langle p, \eta(z, x)\rangle
$$

for all $z \in X$. Hence $X$ is inclosed with respect to $\eta$, i.e., $\eta$-closed. This completes the proof of the theorem.

So far we have discuss the $\eta$-closedness of $X$ from the internal point of view. Now we consider it from the external point of view. In this sense, we frame the concept of exterior $p$-closedness of $X$ with respect to $\eta$ as follows. 
Definition 2.6. The set $X \subset \mathbb{H}$ is $p$-closed with respect to $\eta: X \times X \rightarrow \mathbb{H}$ in the sense exterior point of view if for every $p \in \mathbb{H}$, there exists a unique $x \in X$ closest to $p+\operatorname{t\eta }(z, x) \in \bar{X}$ for all $z \in X$ and $t \in(0,1)$, where $\bar{X}$ denotes the closure of $X$.

Remark 2.7. If $X$ is closed, we get $\bar{X}=X$. So p-closedness of $X$ with respect to $\eta$ in the sense exterior point of view will be coincide with the closedness of $X$ with respect to $\eta$ in the sense internal point of view given in Definition 2.2. In this case $t=0$.

Corollary 2.8. Let $X$ be any subset of the Hilbert space $\mathbb{H}$. Let $\eta$ : $X \times X \rightarrow \mathbb{H}$ be any map. If for every $p \in \mathbb{H}, X$ is $p$-closed with respect to $\eta$. Then $X$ is $\eta$-closed if and only if the following condition holds: for every $p \in \mathbb{H}$, there exists a unique $x \in X$ such that

$$
\langle p, z-x+\operatorname{t\eta }(z, x)\rangle \leq \frac{1}{2}(\langle x, x\rangle-\langle z, z\rangle)+t\langle z, \eta(z, x)\rangle
$$

for all $z \in X$ and $t \in(0,1)$.

Proof. Let for every $p \in \mathbb{H}$, there exists a unique $x \in X$ such that

$$
\langle p, z-x+t \eta(z, x)\rangle \leq \frac{1}{2}(\langle x, x\rangle-\langle z, z\rangle)+t\langle z, \eta(z, x)\rangle
$$

for all $z \in X$ and $t \in(0,1)$. The above expression can be written as

$$
2 t\langle p-z, \eta(z, x)\rangle \leq 2\langle p, z-x\rangle+\langle x, x\rangle-\langle z, z\rangle
$$

for all $z \in X$ and $t \in(0,1)$, which is condition (ii) of Theorem 2.4. $X$ is $p$-closed with respect to $\eta$, i.e., for every $p \in \mathbb{H}$, there exists a unique $x \in X$ closest to $p+t \eta(z, x) \in \bar{X}$ for all $z \in X$, where $\bar{X}$ denotes the closure of $X$ that means

$$
\|x-p-t \eta(z, x)\| \leq\|z-p-t \eta(z, x)\|
$$

for all $z \in X$ and $t \in(0,1)$, which is condition $(i)$ of Theorem 2.4. Since all the conditions of Theorem 2.4 are satisfied, it is concluded that $X$ is $\eta$-invex. Conversely, $X$ is $\eta$-closed, i.e.,

$$
\langle x, \eta(z, x)\rangle \geq\langle p, \eta(z, x)\rangle
$$

for all $z \in X$. Since $X$ is $p$-closed with respect to $\eta$,

$$
\|x-p-\operatorname{t\eta }(z, x)\| \leq\|z-p-\operatorname{t\eta }(z, x)\|,
$$


for all $z \in X$ and $t \in(0,1)$, i.e.,

$$
\langle x, x\rangle-2\langle p, x\rangle-2 t\langle x, \eta(z, x)\rangle \leq\langle z, z\rangle-2\langle p, z\rangle-2 t\langle z, \eta(z, x)\rangle,
$$

for all $z \in X$ and $t \in(0,1)$. Thus

$$
2 t\langle x, \eta(z, x)\rangle \geq 2 t\langle p, \eta(z, x)\rangle
$$

only if

$$
2 t\langle p-z, \eta(z, x)\rangle \leq 2\langle p, z-x\rangle+\langle x, x\rangle-\langle z, z\rangle
$$

for all $z \in X$ and $t \in(0,1)$, i.e.,

$$
\langle p, z-x+t \eta(z, x)\rangle \leq \frac{1}{2}(\langle x, x\rangle-\langle z, z\rangle)+t\langle z, \eta(z, x)\rangle
$$

for all $z \in X$ and $t \in(0,1)$. This completes the proof.

\section{3. $x^{*}$-Generalized Variational Inequality Problem in Hilbert Space}

Let $X$ be a real Hilbert space and $K \subset X$. Let $K^{\oplus}$ and $K^{\ominus}$ be the positive and negative polar cones of $K$ respectively, defined by

$$
K^{\oplus}=\left\{\xi \in X^{*} \equiv X:\langle\xi, v\rangle \geq 0 \quad \text { for all } v \in X\right\}
$$

and

$$
K^{\ominus}=\left\{\xi \in X^{*} \equiv X:\langle\xi, v\rangle \leq 0 \text { for all } v \in X\right\} .
$$

In this section, we first establish the existence theorem of $x^{*}$-variational inequality in Hilbert space using fixed point theorem, then using it we prove the existence theorem of $x^{*}$-generalized variational inequality in Hilbert space.

Let $X$ be a real Hilbert space. Let $K$ be a nonempty subset of $X$ with its dual $X^{*}=X$. Let $T: K \rightarrow X^{*}$ be a nonlinear operator.

We consider the problem $x^{*}$-generalized variational inequality problem $\left(x^{*}\right.$ GVIP) to:

find $y_{0} \in K$ such that

$$
\left\langle T\left(y_{0}\right), \eta\left(z, y_{0}\right)\right\rangle \geq\left\langle x^{*}, \eta\left(z, y_{0}\right)\right\rangle
$$

for all $z \in K$, where $\eta: K \times K \rightarrow X$ is any vector valued map. 
We prove the existence and uniqueness theorem for the nonlinear generalized variational inequality problem by using Banach Contraction Theorem (see, [5]) which states that a contraction map $\phi: K \rightarrow X$ has a fixed point in $K$, where $X$ is a Hilbert space, $K$ is closed convex cone in $X$.

For our need, we recall the following definitions. Let $X$ be a Hilbert space. Let $K$ be a subset of $X$ with dual $X^{*} \equiv X$.

Definition 3.1. A mapping $T: K \rightarrow X^{*}$ is said to be

(a) strongly monotone with relaxation $c$ if for all $x, y \in K$, there exists a constant $c>0$ such that

$$
\langle T(x)-T(y), x-y\rangle \geq c\|x-y\|^{2},
$$

(b) a contraction with rank $k$ if for all $x, y \in K$, there exists a $k \in(0,1)$ such that

$$
\|T(x)-T(y)\| \leq k\|x-y\|
$$

Theorem 3.2. Let $X$ be a Hilbert space and $K$ be a closed subset of $X$ with its dual $X^{*} \equiv X$. Let $\eta: K \times K \rightarrow X$ be any antisymmetric map. Let $K$ be $\eta$-invex. Let $T: K \rightarrow X^{*} \equiv X$ be a nonlinear operator such that $T(y)$ is strongly monotone with relaxation $c$ for each $y \in K$ and Lipschitzian with rank $k$ satisfying $k^{2}<2 c<k^{2}+1$. Then there is a unique $y_{0} \in K$ such that for any $x^{*} \in X^{*}$,

$$
\left\langle T\left(y_{0}\right), \eta\left(z, y_{0}\right)\right\rangle \geq\left\langle x^{*}, \eta\left(z, y_{0}\right)\right\rangle
$$

for all $z \in K$, i.e., $T\left(y_{0}\right)-x^{*} \in K^{\oplus}$.

Proof. By Theorem 2.5, $K$ is closed and $\eta$-invex in $X$. So $K$ is $\eta$-closed in $X$, i.e., for every $y \in K$, there is a unique $x \in K$ closest to $p=y-T(y)+x^{*} \in X$ with respect to $\eta$ such that

$$
\langle x, \eta(z, x)\rangle \geq\langle p, \eta(z, x)\rangle
$$

for all $z \in K$, implying

$$
\langle x, \eta(z, x)\rangle \geq\left\langle y-T(y)+x^{*}, \eta(z, x)\right\rangle
$$

for all $z \in K$, i.e.,

$$
\left\langle x-y+T(y)-x^{*}, \eta(z, x)\right\rangle \geq 0
$$


for all $z \in K$. Let $\theta$ be the correspondence between $y$ and $x$ defined by the rule

$$
\theta(y)=x
$$

Let $y_{1}, y_{2} \in K$ be such that $y_{1} \neq y_{2}$ with $\theta\left(y_{1}\right)=x_{1}$ and $\theta\left(y_{2}\right)=x_{2}$. Putting $y=y_{1}, x=\theta\left(y_{1}\right)$ and $z=\theta\left(y_{2}\right)$ in (3.1), we get

$$
\left\langle\theta\left(y_{1}\right)-y_{1}+T\left(y_{1}\right)-x^{*}, \eta\left(\theta\left(y_{2}\right), \theta\left(y_{1}\right)\right)\right\rangle \geq 0 .
$$

Since $\eta$ is antisymmetric on $K$, we have

$$
\eta(v, u)=-\eta(u, v)
$$

for all $u, v \in K$. From the above equation, we get

$$
\left\langle-\theta\left(y_{1}\right)+y_{1}-T\left(y_{1}\right)+x^{*}, \eta\left(\theta\left(y_{1}\right), \theta\left(y_{2}\right)\right)\right\rangle \geq 0 .
$$

Again putting $y=y_{2}, x=\theta\left(y_{2}\right)$ and $z=\theta\left(y_{1}\right)$ in (3.1), we get

$$
\left\langle\theta\left(y_{2}\right)-y_{2}+T\left(y_{2}\right)-x^{*}, \eta\left(\theta\left(y_{1}\right), \theta\left(y_{2}\right)\right)\right\rangle \geq 0 .
$$

Adding the above two equation, we get

$$
\left\langle\theta\left(y_{2}\right)-\theta\left(y_{1}\right)-y_{2}+y_{1}+T\left(y_{2}\right)-T\left(y_{1}\right), \eta\left(\theta\left(y_{1}\right), \theta\left(y_{2}\right)\right)\right\rangle \geq 0 .
$$

Taking $K$ is $\eta$-invex set, we have

$$
\theta\left(y_{1}\right)=\theta\left(y_{2}\right)+t \eta\left(\theta\left(y_{1}\right), \theta\left(y_{2}\right)\right)
$$

for $t \in[0,1]$. At $t=1$, we get

$$
\theta\left(y_{1}\right)-\theta\left(y_{2}\right)=\eta\left(\theta\left(y_{1}\right), \theta\left(y_{2}\right)\right) .
$$

Hence

$$
\begin{aligned}
\left\langle y_{1}-y_{2}+T\left(y_{2}\right)-T\left(y_{1}\right), \eta\left(\theta\left(y_{1}\right), \theta\left(y_{2}\right)\right)\right\rangle & \geq\left\langle\theta\left(y_{1}\right)-\theta\left(y_{2}\right), \eta\left(\theta\left(y_{1}\right), \theta\left(y_{2}\right)\right)\right\rangle \\
& =\left\langle\theta\left(y_{1}\right)-\theta\left(y_{2}\right), \theta\left(y_{1}\right)-\theta\left(y_{2}\right)\right\rangle \\
& =\left\|\theta\left(y_{1}\right)-\theta\left(y_{2}\right)\right\|^{2}
\end{aligned}
$$

i.e.,

$$
\begin{aligned}
\left\|\theta\left(y_{1}\right)-\theta\left(y_{2}\right)\right\|^{2} & \leq\left\langle y_{1}-y_{2}+T\left(y_{2}\right)-T\left(y_{1}\right), \eta\left(\theta\left(y_{1}\right), \theta\left(y_{2}\right)\right)\right\rangle \\
& \leq\left\|y_{1}-y_{2}+T\left(y_{2}\right)-T\left(y_{1}\right)\right\|\left\|\eta\left(\theta\left(y_{1}\right), \theta\left(y_{2}\right)\right)\right\|
\end{aligned}
$$




$$
=\left\|y_{1}-y_{2}+T\left(y_{2}\right)-T\left(y_{1}\right)\right\|\left\|\theta\left(y_{1}\right)-\theta\left(y_{2}\right)\right\|,
$$

implying

$$
\left\|\theta\left(y_{1}\right)-\theta\left(y_{2}\right)\right\| \leq\left\|y_{1}-y_{2}+T\left(y_{2}\right)-T\left(y_{1}\right)\right\|=\left\|\left(T\left(y_{1}\right)-T\left(y_{2}\right)\right)-\left(y_{1}-y_{2}\right)\right\| .
$$

Since $T$ is $T(y)$ is strongly monotone with relaxation $c$ for each $y \in K$ and Lipschitzian with rank $k$ satisfying $k^{2}<2 c<k^{2}+1$, we have

$$
\left\|T\left(y_{1}\right)-T\left(y_{2}\right)\right\| \leq k\left\|y_{1}-y_{2}\right\|
$$

and

$$
\left\langle T\left(y_{1}\right)-T\left(y_{2}\right), y_{1}-y_{2}\right\rangle \geq c\left\|y_{1}-y_{2}\right\|^{2}
$$

Thus

$$
\begin{aligned}
\left\|\theta\left(y_{1}\right)-\theta\left(y_{2}\right)\right\|^{2}= & \left\|\left(T\left(y_{1}\right)-T\left(y_{2}\right)\right)-\left(y_{1}-y_{2}\right)\right\|^{2} \\
= & \left\|T\left(y_{1}\right)-T\left(y_{2}\right)\right\|^{2}+\left\|y_{1}-y_{2}\right\|^{2} \\
& -2\left\langle T\left(y_{1}\right)-T\left(y_{2}\right), y_{1}-y_{2}\right\rangle \\
\leq & k^{2}\left\|y_{1}-y_{2}\right\|^{2}+\left\|y_{1}-y_{2}\right\|^{2}-2 c\left\|y_{1}-y_{2}\right\|^{2} \\
= & \left(k^{2}+1-2 c\right)\left\|y_{1}-y_{2}\right\|^{2} \\
= & \lambda^{2}\left\|y_{1}-y_{2}\right\|^{2},
\end{aligned}
$$

where $\lambda^{2}=k^{2}+1-2 c \in(0,1)$ since $k^{2}<2 c<k^{2}+1$, implying $\lambda \in(0,1)$. Hence

$$
\left\|\theta\left(y_{1}\right)-\theta\left(y_{2}\right)\right\| \leq \lambda\left\|y_{1}-y_{2}\right\|,
$$

implies that $\theta$ is a contraction in $K$. From Banach Contraction Theorem we conclude that $\theta$ has a fixed point in $K$, say $y_{0}$. Since $\theta\left(y_{0}\right)=y_{0}$, putting $y=y_{0}$ and $x=\theta\left(y_{0}\right)$ in (3.1), we get

$$
\left\langle T\left(y_{0}\right)-x^{*}, \eta\left(z, y_{0}\right)\right\rangle \geq 0
$$

for all $z \in K$, i.e.,

$$
\left\langle T\left(y_{0}\right), \eta\left(z, y_{0}\right)\right\rangle \geq\left\langle x^{*}, \eta\left(z, y_{0}\right)\right\rangle
$$

for all $z \in K$, i.e., the unique $y_{0}$ solves $\left(x^{*}-G V I P\right)$ and $T\left(y_{0}\right)-x^{*} \in K^{\oplus}$. This completes the proof of the theorem. 


\section{Generalized Banach Contraction Principle}

We consider the problem $x^{*}$-generalized complementarity problem $\left(x^{*}\right.$-GCP) to:

find $y_{0} \in K$ such that

$$
T\left(y_{0}\right)-x^{*} \in K^{\oplus}
$$

and

$$
\left\langle T\left(y_{0}\right), \eta\left(z, y_{0}\right)\right\rangle=\left\langle x^{*}, \eta\left(z, y_{0}\right)\right\rangle
$$

for all $z \in K$, where $\eta: K \times K \rightarrow X$ is any vector valued map.

For our need we recall the result studied by Nanda and Nanda [9] using Banach contraction principle.

Theorem 4.1. [9] Let $X$ be a Hilbert space. Let $K$ be a subset of $X$ with its dual $X^{*} \equiv X$ and $T: K \rightarrow X$ be a strongly monotone and Lipschitzian with

$$
k^{2}<2 c<k^{2}+1 .
$$

Then there is a unique $y_{0}$ such that

$$
y_{0} \in K, \quad T\left(y_{0}\right) \in K^{\oplus} \quad \text { and } \quad\left\langle T\left(y_{0}\right), y_{0}\right\rangle=0 .
$$

To prove the existence theorem of generalized Banach contraction principle, we recall the definition of condition $C_{0}$ introduced by Behera and Das [1].

Definition 4.2. (Condition $C_{0}$ ) [1] Let $K$ be any $\eta$-invex subset of the vector space $X$, where $\eta: K \times K \rightarrow X$ be a vector valued mapping. $\eta$ is said to satisfy condition $C_{0}$ if for all $u, v \in K$ and for all $t \in(0,1)$, we have

(a) $\eta(u, z)+\eta(z, u)=0$, where $z=v+\eta(u, v)$,

(b) $\eta(v+t \eta(u, v), v)=-t \eta(u, v) \in K$.

We extend the Theorem 4.1 as follows.

Theorem 4.3. (Generalized Banach contraction principle) Let $X$ be a Hilbert space and $K$ be a closed subset of $X$ with its dual $X^{*} \equiv X$. Let $\eta: K \times K \rightarrow X$ be any antisymmetric map. Let $K$ be $\eta$-invex, where $\eta$ satisfies condition $C_{0}$. Let $T: K \rightarrow X^{*} \equiv X$ be a nonlinear operator such that $T(y)$ is strongly monotone with relaxation $c$ for each $y \in K$ and Lipschitzian with rank 
$k$ satisfying $k^{2}<2 c<k^{2}+1$. Then there is a unique $y_{0} \in K$ such that for any $x^{*} \in X^{*}, T\left(y_{0}\right)-x^{*} \in K^{\oplus}$ and

$$
\left\langle T\left(y_{0}\right), \eta\left(z, y_{0}\right)\right\rangle=\left\langle x^{*}, \eta\left(z, y_{0}\right)\right\rangle
$$

for all $z \in K$, i.e., $y_{0}$ solves $\left(x^{*}-G C P\right)$.

Proof. Since all the conditions of Theorem 3.2 are satisfied, there exists a unique $y_{0} \in K$ such that for any $x^{*} \in X^{*}$,

$$
\left\langle T\left(y_{0}\right), \eta\left(z, y_{0}\right)\right\rangle \geq\left\langle x^{*}, \eta\left(z, y_{0}\right)\right\rangle
$$

for all $z \in K$, i.e., $T\left(y_{0}\right)-x^{*} \in K^{\oplus}$. Since $K$ is $\eta$-invex set and $\eta$ satisfies condition $C_{0}$, we get

$$
\eta\left(y_{0}+t \eta\left(z, y_{0}\right), y_{0}\right)=-t \eta\left(z, y_{0}\right)
$$

for all $z \in K$. Replacing $z$ by $y_{0}+t \eta\left(z, y_{0}\right)$ in (4.1) and using condition $C_{0}$, we get

$$
\left\langle T\left(y_{0}\right), \eta\left(z, y_{0}\right)\right\rangle \leq\left\langle x^{*}, \eta\left(z, y_{0}\right)\right\rangle
$$

for all $z \in K$ which together with (4.1) gives

$$
\left\langle T\left(y_{0}\right), \eta\left(z, y_{0}\right)\right\rangle=\left\langle x^{*}, \eta\left(z, y_{0}\right)\right\rangle
$$

for all $z \in K$. Hence $y_{0}$ solves $\left(x^{*}-G C P\right)$. This completes the proof of the theorem.

Remark 4.4. If $\eta(x, u)=x-u$ and $x^{*}=0$, then the result of Theorem 4.3 coincides with the result studied by Nanda and Nanda [9].

\section{Acknowledgement}

The authors are very thankful to the two referees, whose helpful and essential corrections greatly improved an earlier version of the manuscript.

\section{References}

[1] A. Behera and P. K. Das, Variational Inequality Problems in $H$-spaces, International Journal of Mathematics and Mathematical Sciences, Article ID. $\mathbf{7 8 5 4 5}$ (2006) 1-18. 
[2] M. Chipot, Variational Inequalities and Flow in Porus Media, Appl. Math. Sc., 52, Springer-Verlag (1984).

[3] R. W. Cottle, F. Giannessi, J.L.Lions, Variational inequality and Complementarity Problems-Theory and Application, John Wiley and Sons (1980).

[4] P. K. Das, S. K. Mohanta, Generalized Vector Variational Inequality Problem, Generalized Vector Complementarity Problem in Hilbert Spaces, Riemannian $n$-Manifold, $\mathbb{S}^{n}$ and Ordered Topological Vector Spaces: A Study using Fixed Point Theorem and Homotopy Function, Advances in Nonlinear Variational Inequalities, 12(2) (2009), 37-47.

[5] C. Goffman and G. Pedrick: A first course in functional analysis, PrenticeHall, New Delhi (1974).

[6] M. A. Hanson, On Sufficiency of the Kuhn-Tucker Conditions, Journal of Mathematical Analysis and Applications, 80 (1981), 545-550.

[7] G. Isac, Complementarity Problem : Lecture Notes in Mathematics, 1528, Springer-Verlag, New York (1991).

[8] J. L. Lions, G. Stampacchia, Variational inequality,Comm. Pure. Appl. Math., 48(1) (1967), 493-519.

[9] S. Nanda and S. Nanda, A nonlinear complementarity problem in mathematical programming in Hilbert space, Bulletin of Australian Mathematical Society, 20 (1979), 233-236.

[10] G. Stampacchia, Formes bilineaires coercitives sur les ensembles convexes, Comptes rendus hebdomadaires des séances de l'Académie des sciences, 258 (1964), 4413-4416. 
